\title{
THE ESTIMATION OF THE RADIATION DOSE TO THE LEFT LUNG FOR VARIOUS MODES OF CONFORMAL RADIATION TREATMENT OF THE BREAST CANCER PATIENTS
}

\section{L.I. Korytova1, E.A. Maslyukova1, A.V. Bondarenko ${ }^{1 *}$, O.V. Korytov², E.M. Muravnik}

${ }^{1}$ Russian Research Center of Radiology and Surgical Technologies, Ministry of Healthcare of Russia, Saint-Petersburg, Russia

${ }^{2}$ City Polyclinic №26, Saint-Petersburg, Russia

\begin{abstract}
Lowest dose detection per ipsilateral lung versus various dosimetric irradiation plans for the left mammary gland is investigated. The study involves dosimetric radiotherapeutic (RT) plans of 20 female patients with left BC (breast cancer). Pre-irradiation preparation included 3 sessions of CT scan: patient in standard dorsal position with tidal respiration (STR), in dorsal position with controlled breathhold on top inspiration (DBH) and in prone position with tidal respiration (PTR). Three CT-sessions were followed by $3 D$-plan dosimetric calculations. Dose-volumetric measures for organs at risk $(O A R)$ were assessed for every irradiation option. Contoured left lung volume in all studied variants varied within $757.1 \mathrm{~cm}^{3}-2923.8 \mathrm{~cm}^{3}$, mean volume $1751.6 \mathrm{~cm}^{3}$. The best values, such as $V_{25 l u n g}\left(\right.$ when $\alpha / \beta=3.1$ ) and $V_{28 l u n g}(w h e n ~ \alpha / \beta=9$ ), average doses per ipsilateral lung were received using the PTR method ( $V_{25 l u n g}(\alpha / \beta=3.1)-10.19 \%$, $V_{28 l u n g}(\alpha / \beta=9)-9.19 \%$; Dmean lung $\left.7.42 \mathrm{~Gy}\right)$ versus the $S T R$ method $\left(V_{25 l u n g}\right.$ $(\alpha / \beta=3.1)-20.72 \%$, $V_{28 l u n g}(\alpha / \beta=9)-19.6 \%$; D mean $\left.10.42 \mathrm{~Gy}\right)$ and DBH-position $\left(V_{25 \text { lung }}(\alpha / \beta=3.1)-20.17 \%\right.$, $V_{28 l u n g}$ $(\alpha / \beta=9)-19.01 \%$; Dmean lung $10.11 \mathrm{~Gy})$ included in $M G$ volume and axillary $L N$ with $V_{25}$ lung $(\alpha / \beta=3.1)-P=0.00000^{* * *}$, $V_{28 \text { lung }}(\alpha / \beta=9)-P=0.00000^{* *} ; D$ mean $-p=0.00002^{* *}$. No preferences in dosimetry were detected for the addition of supraclavicular and infraclavicular lymph nodes $(L N)$ in irradiation volume using STR and DBH methods: DBH $\left(V_{25 l u n g}(\alpha / \beta=3.1)-21.49 \%, V_{28 l u n g}(\alpha / \beta=9)-20.17 \%\right.$; $D_{\text {mean lung } 10.85}$ Gy) versus $S T R$ method $\left(V_{25 l u n g}(\alpha / \beta=3.1)-\right.$ $23.07 \%$, $\left.V_{28 \text { lung }}(\alpha / \beta=9)-21.64 \% ; D_{\text {mean lung } 11.72} \mathrm{~Gy}\right)$. $V_{25 \text { lung }}(\alpha / \beta=3.1)-p=0.438, V_{28 l u n g}(\alpha / \beta=9)-p=0.461 ; D_{\text {mean lung }}$ $p=0.2964$. Based on our investigation, the lowest doses per ipsilateral lung were received in prone position with tidal respiration including axillary lymph nodes $(L N)$ in $M G$ volume. These findings were associated with the results of few international studies. No statistically significant difference in left lung radiation exposure was detected during the comparison of STR and DBH methods with the additional irradiation of supraclavicular and infraclavicular $L N$.
\end{abstract}

Key words: Breast cancer, radiotherapy, heart, left anterior descending coronary artery (LAD), controlled breathhold

DOI: $10.21175 /$ RadProc.2017.37

\section{INTRODUCTION}

Due to the growing public awareness, increased number of screening programs, development of imaging techniques and more efficient therapy, there can be observed earlier BR detection and prolonged lifespan; that explains the rising interest in reduced RT toxicity.

Radiation-induced damages of normal tissues are the inevitable corollary of radiation (R) exposure. Incidence and severity of radiation-induced damages depend on multiple factors: irradiation technique, single and integral dose values, individual radiosensitivity, comorbidity, etc. The progression of acute and chronic postradiation complications correlates with the total boost dose (TBD), fraction dose and the volume of exposed lung tissue $[1,2,3]$.

Radial pulmonitis includes a wide range of clinical signs and symptoms. Manifestations vary from incidental radiological findings to respiratory gross lesions in patients with expressed clinical manifestations. The symptoms of acute radial pulmonitis are routinely expressed in 2-3 months after radiotherapy, but they can also appear in a month or 6 months after treatment termination $[1,4,5]$.

Radial pulmonitis is the inflammatory condition that can be completely resolved (independently or affected by antibacterial and hormone therapy) or progress to nonreversible fibrosis. In case of pulmonary fibrosis progression, lung function test values may decrease. In the setting of minor pulmonary lesion (small area), the adjacent lung areas can compensate respiratory function and prevent the progression of serious clinical changes [6].

The process of lung fibrosis lasts for 6-24 months and usually becomes stable within 2 years. The progression of lung fibrosis may be symptom-free or be characterized by the signs of respiratory impairment depending on the area of lung tissues exposed to

*zaharikispb@mail.ru 
fibrosis and compensation abilities of unexposed lung tissues. Lung tissue becomes functionally incomplete and may potentially cause the progression of pulmonary hypertension, which is associated with heart failure $[7,8]$.

Clinical observations highlight the necessity to avoid the dosage per total lung volume of above $20 \mathrm{~Gy}$ if it is not certain that unexposed lung shall compensate respiratory function. Goldman et al., in their work [9] on 3D conformal radiotherapy with left lung limitation $\mathrm{V}_{20} \leq 30 \%$ in 88 patients at the early stages of left BC, reported on the single case of acute pulmonitis, which led to the prescription of corticosteroid and hormone therapy. Moreover, some works indicate the relation between the progression of postradiation respiratory complications and the age of a patient [9].
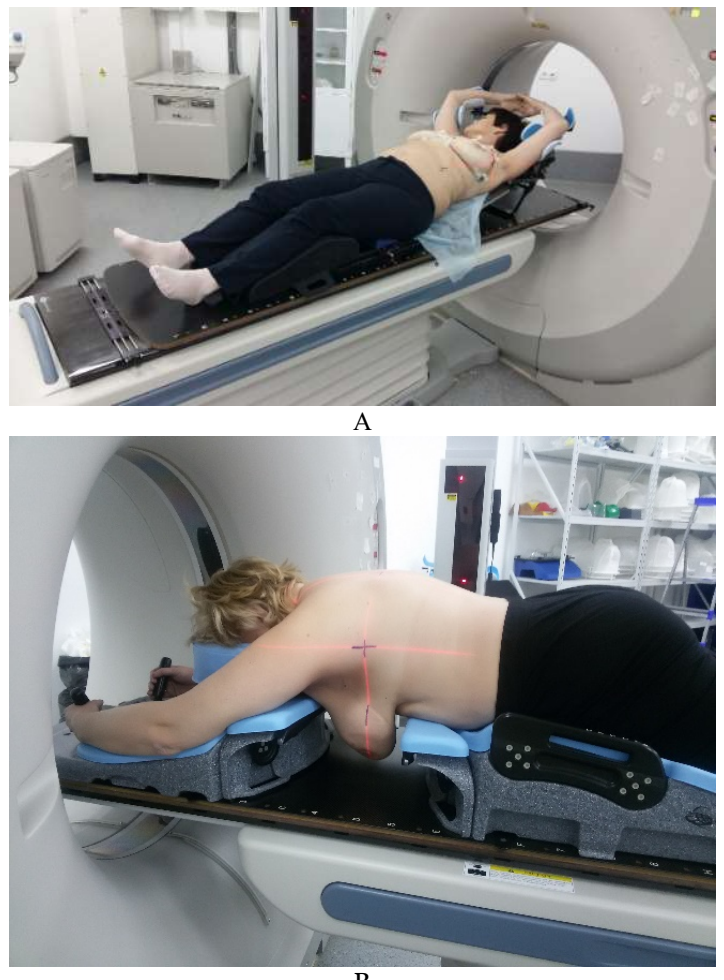

B

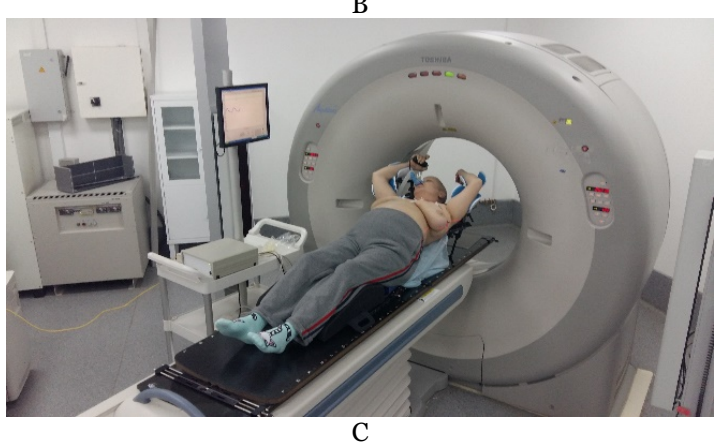

Figure 1. Patient positioning during pre-irradiation preparation: A - dorsal position with tidal respiration; $\mathrm{B}$ - prone position; $\mathrm{C}$ - dorsal position with controlled breathhold on top inspiration

Thus, the reduction of radiation exposure per lung in the course of radiotherapy in patients with BC 182 represents the relevant objective for clinical radiotherapists [10].

The purpose of this study resides in the prospective comparison of three various options of whole MG irradiation with/without supraclavicular and infraclavicular LN: standard dorsal position with tidal respiration (STR), dorsal position with controlled breathhold on top inspiration (DBH) and prone position with tidal respiration (PTR). The aim is to evaluate the impact of the given methods on the dosimetric indices of the radiation exposure of the left lung.

\section{MATERIALS AND TECHNIQUES}

RT plans of 20 patients with left BC were prepared for analysis in the Federal state budgetary institution "Russian Research Center of Radiology and Surgical Technologies" of the Ministry of Health of the Russian Federation. 8 dosimetric plans were calculated for every studied case based on three CT-scan sessions:

1. In dorsal position using an individually modified breast-board with tidal respiration with/without the inclusion of supraclavicular and infraclavicular LN (plan 1/2), Fig. 1A.

2. In prone position using a prone-board, without/with the inclusion of axillary LN (plan 3/4), Fig. 1B.

3. In dorsal position using an individually modified breast-board with breathhold, with/without the inclusion of supraclavicular and infraclavicular LN (ptv $1.0 \mathrm{~cm}$, plan 5/6 and ptv $0.5 \mathrm{~cm}$, plan $7 / 8$ ), Fig. 1C.

ABC device (Elekta, Sweden) was used for the active control over breathhold on top inspiration, its construction comprised a personal computer with specific software installed, spirometer, respiratory tube (for patient's breathing during the entire procedure of $\mathrm{RT}$ ). After the passage of threshold air volume through the spirometer (set individually in the training course), the valve built in respiratory tube interrupts the respiratory cycle at the given value. Fraction dose is 3 Gy, physical total boost dose (TBDphys) per mammary gland - 42 Gy., while in the case of supraclavicular and infraclavicular lymph node inclusion, LN TBDphys is 39 Gy. Prestrictions on critical structures (spinal cord, ipsilateral lung, heart) were given in accordance with standard RTOG recommendations. Therefore, considering the application of average fractionation, the reassessment of biologically effective doses related to radioreaction and complication manifestations on the part of lungs based $\alpha / \beta$ is performed by the following formula:

$$
\frac{\mathcal{D}_{1}}{\mathcal{D}_{2}}=\frac{d_{2}+\frac{a}{b}}{d_{1}+\frac{a}{b}}
$$

$D_{1}$ - total dose in standard fractionation mode, $D_{2}$ - isoeffective dose in average fractionation mode, $d_{1}$ - fraction dose in standard fractionation mode, $\mathrm{d}_{1}$ - fraction dose in average fractionation mode.

V30 in standard fractionation corresponded to early radioreactions (pulmonitis, $\alpha / \beta=9$ ) $-V_{28 \text { pulm, for }}$ complications (pulmonary fibrosis, $\alpha / \beta=3.1$ ) $-V_{25 p u l m}$. 
CT findings were transferred to the station of dosimetric planning (Xio, Elekta). Lungs were contoured using the function of the automatic countering program Monaco (Elekta). All the stages of pre-irradiation preparation were performed by the same team, which included a topometrist, radiotherapist and physicist in medicine (Fig.2).

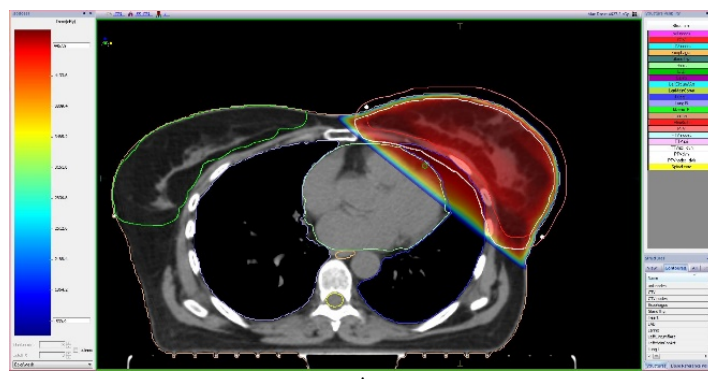

A

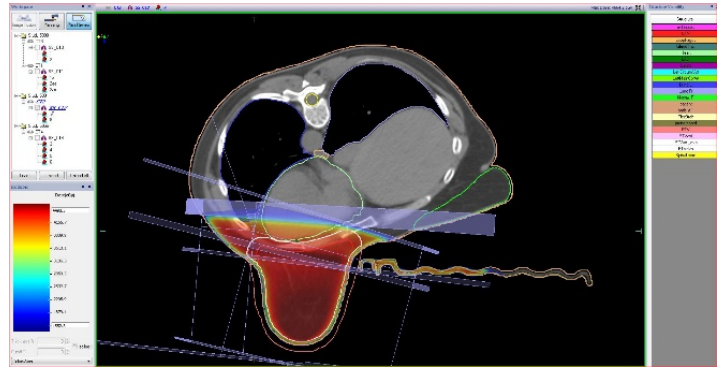

B

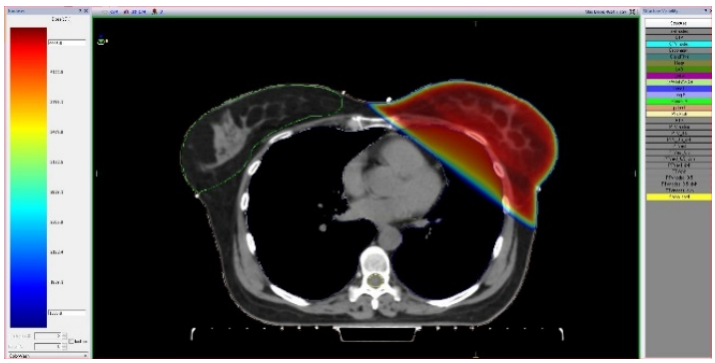

C

Figure 2. Dosimetric distribution using different methods: A - dorsal position with tidal respiration; B - prone position; $\mathrm{C}$ - dorsal position with controlled breathhold on top inspiration

Average fractionation was applied. The fraction dose was $3 \mathrm{~Gy}$, whereas TBD per mammary gland was 42 Gy. DVH (dose-volume histogram) was generated for all contoured structures within all plans per every patient. The following values were calculated for ipsilateral lung: exposed volume percent $\geq 28$ Gy ( $V_{28 l u n g}$ when $\alpha / \beta=9$ ) for early pulmonitis, and exposed volume percent $\geq 25$ Gy $\left(V_{25 l u n g} \alpha / \beta=3.1\right)$ for late complications - pulmonary fibrosis.

Statistical analysis was performed using the program STATISTICA 12.

Descriptive statistics was used for dose-volumetric parameters using the analysis of variance (ANOVA), focused on correlation search among experimental data via diversity investigation of differences in average values, which enabled the comparison between three and more groups. For all statistical tests, $\mathrm{P}<0.05$ was statistically significant.

\section{RESULTS}

The comparison of $\mathrm{D}_{\text {mean lung }}$ revealed that the best results were achieved in PTR group, the worst - in STR position (Table 1, Fig. 3).

Table 1. The comparison of $\mathrm{D}_{\text {mean lung }}$

\begin{tabular}{|c|c|c|c|c|c|c|c|}
\hline Group & № & $\begin{array}{l}\text { Mean } \\
\text { Dose, } \\
\text { cGy }\end{array}$ & $\begin{array}{l}\text { Median, } \\
\text { cGy }\end{array}$ & \begin{tabular}{|l|} 
MinD \\
ose, \\
cGy
\end{tabular} & $\begin{array}{l}\text { MaxDos } \\
\text { e, cGy }\end{array}$ & StdDev & $\begin{array}{l}\text { Stand } \\
\text { ard }\end{array}$ \\
\hline All series CT & 160 & 906.94 & 892.8 & 122.4 & 1674.3 & 290.76 & 25.9 \\
\hline $\mathrm{STR}+\mathrm{LN}$ & 20 & 1172.07 & 1163.6 & 793.5 & 1615.0 & 218.97 & 54.74 \\
\hline STR-LN & 20 & 1040.42 & 1023.7 & 707.9 & 1394.8 & 173.89 & 43.47 \\
\hline PTR-LNAx & 20 & 412.11 & 419.8 & 122.4 & 603.5 & 144.41 & 37.28 \\
\hline PTR+LNAx & 20 & 742.0 & 713.9 & 535.4 & 901.9 & 119.65 & 30.89 \\
\hline $\begin{array}{l}\text { DBH+LN } \\
\text { ptv } 1.0 \mathrm{~cm}\end{array}$ & 20 & 1085.53 & 1008.5 & 792.5 & 1674.3 & 241.17 & 60.29 \\
\hline $\begin{array}{l}\text { DBH-LN } \\
\text { ptv } 1.0 \mathrm{~cm}\end{array}$ & 20 & 1011.33 & 921.8 & 795.1 & 1523.3 & 202.97 & 50.74 \\
\hline $\begin{array}{l}\mathrm{DBH}+\mathrm{LN} \\
\text { ptv } 0.5 \mathrm{~cm}\end{array}$ & 20 & 905.86 & 845.4 & 696.4 & 1409.0 & 208.97 & 52.24 \\
\hline $\begin{array}{l}\text { DBH-LN } \\
\text { ptv } 0.5 \mathrm{~cm}\end{array}$ & 20 & 844.96 & 770.95 & 648.4 & 1309.6 & 186.26 & 46.56 \\
\hline
\end{tabular}

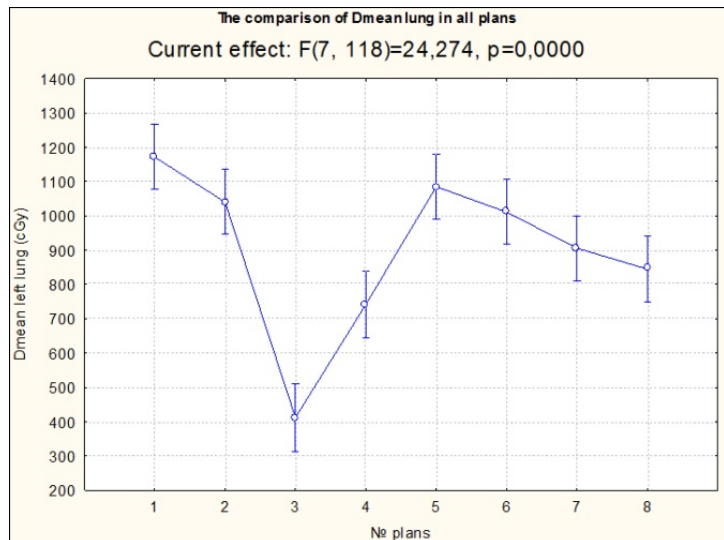

Figure 3. The comparison of $\mathrm{D}_{\text {mean lung in all plans }}$

The average lung volume in dorsal position with tidal respiration was $1236.6 \mathrm{~cm}^{3}$, in prone position $1233.6 \mathrm{~cm}^{3}$, in dorsal position with controlled breathhold - $2251.8 \mathrm{~cm}^{3}$, (Fig. 4).

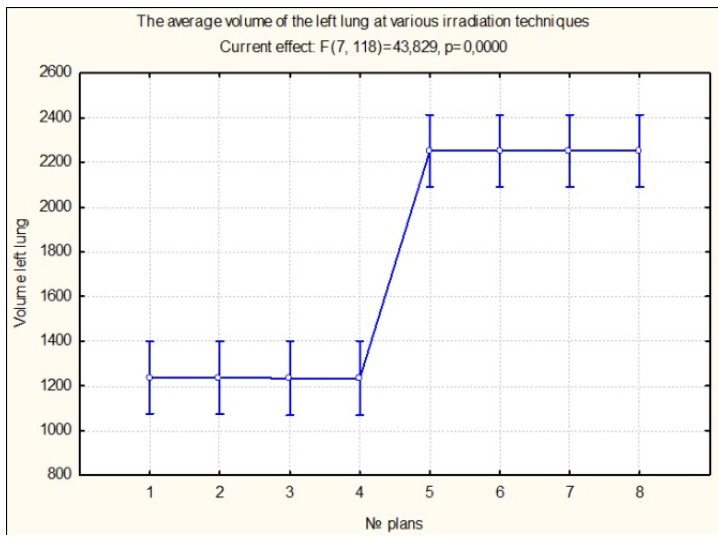

Figure 4. The average volume of the left lung at various irradiation techniques 
Comparison of STR, DBH and PTR plans with exposed MG and axillary $\mathrm{LN}$, and the minimum values of $\mathrm{D}_{\text {mean }}$ lung were registered in prone position $\left(\mathrm{p}=0.00002^{* *}\right)$, (Fig. 5, Table 2).

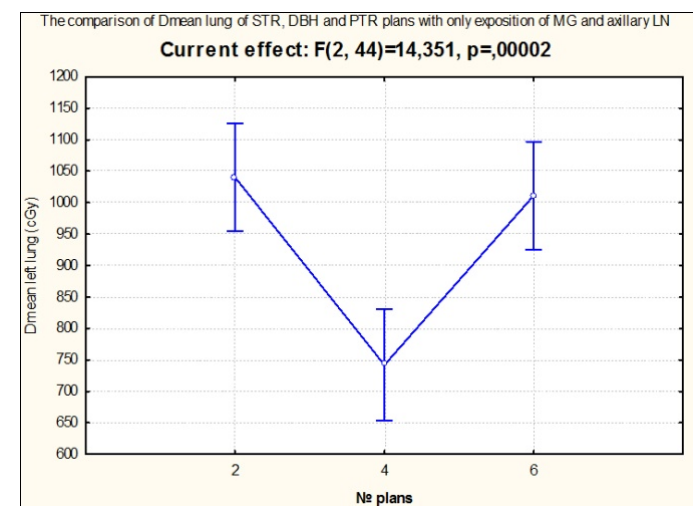

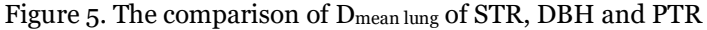
plans with only exposition of MG and axillary LN

Table 2. The comparison of Dmean lung and $\mathrm{V}_{28}$ lung of STR, DBH and PTR plans with only the exposition of MG and axillary LN

\begin{tabular}{|l|c|c|c|c|}
\hline & STR & PTR & DBH & P-value \\
\hline Dmean lung, Gy & 10.42 & 7.42 & 10.11 & $\mathrm{P}=$ o.00002 $^{* *}$ \\
\hline $\mathrm{V}_{28 \text { lung }(\alpha / \beta=9)}$ & 19.60 & 9.19 & 19.01 & $\mathrm{P}=$ o.00000 $^{* *}$ \\
\hline $\mathrm{V}_{25 \text { lung }(\alpha / \beta=3)}$ & 20.72 & 10.19 & 20.17 & $\mathrm{P}=$ o.00000 $^{* *}$ \\
\hline
\end{tabular}

Dosage analysis per lung in STR, DBH and PTR plans, with the inclusion of supraclavicular and infraclavicular LN in exposed area (comparison of STR and DBH methods), did not register significant differences $(\mathrm{p}=0.296)$, (Fig. 6, Table 3).

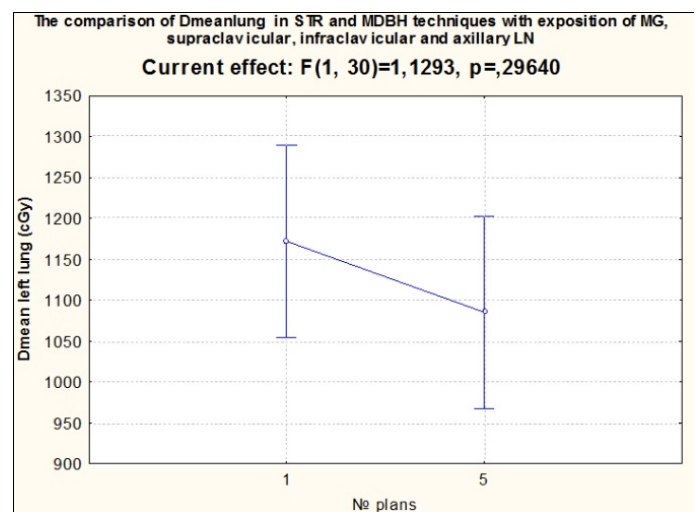

Figure 6. The comparison of Dmean lung in STR and DBH techniques with the exposition of MG, supraclavicular, infraclavicular and axillary LN

Table 3. The comparison of Dmean lung and $\mathrm{V}_{28}$

\begin{tabular}{|c|c|c|c|}
\hline & STR & DBH & P-value \\
\hline$D_{\text {mean lung, Gy }}$ & 11.72 & 10.85 & $\mathrm{P}=0.2964$ \\
\hline $\mathrm{V}_{28 \text { lung }(\alpha / \beta=9)}$ & 21.64 & 20.17 & $\mathrm{P}=0.461$ \\
\hline $\mathrm{V}_{25}$ lung $(\alpha / \beta=3)$ & 23.07 & 21.49 & $\mathrm{P}=0.438$ \\
\hline
\end{tabular}

The same pattern was observed during the estimation of $V_{25}(\alpha / \beta=3.1)$ and $V_{28}(\alpha / \beta=9)$ values. The highest doses per $V_{\text {25lung }}(\alpha / \beta=3.1)$ and $V_{28 l u n g}$ $(\alpha / \beta=9)$ of the left lung were received in STR position, the least - in PTR position, without the exposition of supraclavicular and infraclavicular LN $(\mathrm{p}<0.05)$ (Table 2).

Dosage analysis per left lung in STR, DBH and PTR plans with the exposure of supraclavicular and infraclavicular LN (comparison of STR and DBH methods) did not reveal significant differences (p>0.05), (Table 3). Substantial irradiation of supraclavicular and infraclavicular $\mathrm{LN}$ in prone position does not seem technically possible.

Comparison of the given indices: $\mathrm{D}_{\text {mean lung }}, \mathrm{V}_{28 \text { lung }}$ $(\alpha / \beta=9)$ and $V_{25 l u n g}(\alpha / \beta=3.1)$ of the left lung revealed the following patterns - the prone position with tidal respiration including only exposed MG and axillary $\mathrm{LN}$ is the most preferable in the context of lung irradiation reduction; the analysis of $D_{\text {mean lung, }} V_{28 l u n g}(\alpha / \beta=9)$ and $\mathrm{V}_{25 \text { lung }}(\alpha / \beta=3.1)$ values using STR and DBH methods, with the inclusion of supraclavicular and infraclavicular LN does not reveal any benefits among these methods based on the given indices.

\section{Discussion}

Previously diagnosed diseases, young BC patients and certain achievements of pharmacological treatment led to the increase of life span. Development and implementation of advanced techniques in practical healthcare associated with the reduction of radiation exposure of OAR have a great importance at the present days. The purpose of this study was focused on the analysis of different methods and associated impact of RT in BC on the distribution of dosevolumetric parameters for critical structures. Statistically significant dose reductions per ipsilateral lung were detected in prone position. However, these benefits can only be used for mammary gland irradiation at the early stages with unexposed supraclavicular and infraclavicular LN. Thus, it is applicable for the limited category of patient population. Radiation doses per lung with exposed supraclavicular and infraclavicular $\mathrm{LN}$ in dorsal position with tidal respiration or breathhold were similar. In addition, based on our previous research, it was outlined that cardiac radiation exposure in prone position was the worst [4]. Thus, patients' prone positioning is more suitable for right-sided patients, so that in this case heart is not exposed to irradiation, and exposure reduction per lung is beneficial.

The impediments to prone-positioned RT may include prolonged patient's pre-irradiation preparation, complications associated with the field supply via collateral mammary gland, multisegment supply aimed to avoid large ionization maximum (which in its turn leads to the significantly increased duration of RT session and, correspondingly, the reduction of linear accelerator capacity), technical incapacity of supraclavicular and infraclavicular LN irradiation, and also difficulties in position repeatability from session to session, which is noted by many authors. 


\section{CONCLUSION}

Prone position may improve dose-volumetric distribution per ipsilateral lung and thus reduce the development risk for pulmonary early reactions and late complications.

Ideal candidates for RT in prone position are represented by the patients with the right-sided lesion of mammary gland, in the absence of indications for supraclavicular and infraclavicular LN exposure.

\section{REFERENCES}

1. L. Bissoli, V. Di Francesco, F. Valbusa et al., "A case of bronchiolitis obliterans organising pneumoniae (BOOP) after nine months post-operative irradiation for breast cancer," Age Ageing, vol. 37, no. 2, p. 235, Jan. 2008.

DOI: $10.1093 /$ ageing/afno10

PMid: 18349018

2. G. R. Epler, "Post-breast cancer radiotherapy bronchiolitis obliterans organizing pneumonia," Expert. Rev. Respir. Med., vol. 7, no. 2, pp.109-112, Apr. 2013.

DOI: $10.1586 /$ ers.13.1

PMid: 23547987

3. D. E. Stover, F. Milite, M. Zakowski, "A newly recognized syndrome - radiation-related bronchiolitis obliterans and organizing pneumonia. A case report and literature review," Respiration, vol.68, no. 5, pp. 540-544, Oct. 2001.

DOI: $10.1159 / 000050566$ PMid: 11694821

4. А. В Бондаренко, Л. И. Корытова, Е. А. Маслюкова, О. В. Корытов, Е. М. Муравник, "Результаты сравнения лучевой нагрузки на сердце и левую переднюю нисходящую коронарную артерию при разных вариантах облучения рака молочной железы," Опухоли женской репродуктивной системы, т. 12, № 3, стр. 10-16, 2016. (A. V. Bondarenko, L. I. Korytova, E. A. Maslyukova, O. V. Korytov, E. M. Muravnik, "The comparison of the radiation load to the heart and the left anterior descending coronary artery for various models of radiation treatment of the breast cancer patients," Women Reproductive System Tumors, vol. 12, no. 3, pp. 10-16, 2016.)

DOI: 10.17650/1994-4098-2016-12-3-10-16

5. D. P. Penney, P. Rubin, "Specific early fine structural changes in the lung irradiation," Int. J. Radiat. Oncol. Biol. Phys., vol. 2, no. 11-12, pp. 1123-1132, Nov-Dec. 1977.

DOI: 10.1016/0360-3016(77)90119-5

6. P. A. Lind, G. Svane, G. Gagliardi C. Svensson, "Abnormalities by pulmonary regions studied with computer tomography following local or local-regional radiotherapy for breast cancer," Int. J. Radiat. Oncol. Biol. Phys., vol. 43, no. 3, pp. 489-496, Feb. 1999.

DOI: 10.1016/So360-3016(98)oo414-3 PMid: 10078627

7. E. K. Chie, K. H. Shin, D. Y. Kim et al., "Radiation Pneumonitis after Adjuvant Radiotherapy for Breast Cancer: A Volumetric Analysis Using CT Simulator," J. Breast Cancer, vol. 12, no. 2, pp. 73-78, Jun 2009. DOI: 10.4048/jbc.2009.12.2.73

8. L. Pierce, A. Lewin, M. Abdel-Wahab et al., "Early radiation-induced lung injury in a patient with prior diagnosis of bronchiolitis obliterans organizing pneumonitis," J. Natl. Med. Assoc., vol. 100, no. 12, pp. $1474-1476$, Dec. 2008. DOI: 10.1016/Soo27-9684(15)31551-0

9. U. B. Goldman, B. Wennberg, G. Svane, "Reduction of radiation pneumonitis by V2O-constraints in breast cancer," Radiation Oncology, vol. 5, no. 99, pp. 1-6, Oct. 2010.

DOI: $10.1186 / 1748-717 \mathrm{X}-5-99$

PMid: 21034456

PMCid: PMC2987943

10. J. Vikström, M. H. Hjelstuen, I. Mjaaland et al., "Cardiac and pulmonary dose reduction for tangentially irradiated breast cancer, utilizing deep inspiration breath-hold with audio-visual guidance, without compromising target coverage," Acta Oncol., vol. 50, no. 1, pp. 42-50, Jan. 2011.

DOI: 10.3109/0284186X.2010.512923

PMid: 20843181 